\title{
Diet composition and activity level of at risk and metabolically healthy obese American adults
}

\author{
Arlene L. Hankinson ${ }^{1}$, Martha L. Daviglus ${ }^{1}$, Linda Van Horn ${ }^{1}$, Queenie Chan ${ }^{2}$, Ian Brown², \\ Elaine Holmes ${ }^{2}$, Paul Elliott ${ }^{2}$, and Jeremiah Stamler ${ }^{1}$ \\ ${ }^{1}$ Feinberg School of Medicine, Northwestern University, Chicago, IL \\ ${ }^{2}$ Department of Epidemiology and Public Health, Faculty of Medicine, St Mary's Campus, Imperial \\ College, London, UK
}

\section{Abstract}

Obesity often clusters with other major cardiovascular disease risk factors, yet a subset of the obese appears to be protected from these risks. Two obesity phenotypes are described, 1) "metabolically healthy" obese, broadly defined as body mass index (BMI) $\geq 30 \mathrm{~kg} / \mathrm{m}^{2}$ and favorable levels of blood pressure, lipids, and glucose; and 2) "at risk" obese, BMI $\geq 30$ with unfavorable levels of these risk factors. More than $30 \%$ of obese American adults are metabolically healthy. Diet and activity determinants of obesity phenotypes are unclear. We hypothesized that metabolically healthy obese have more favorable behavioral factors, including less adverse diet composition and higher activity levels than at risk obese in the multi-ethnic group of 775 obese American adults ages 40-59 years from the International Population Study on Macro/Micronutrients and Blood Pressure (INTERMAP) cohort. In gender stratified analyses, mean values for diet composition and activity behavior variables, adjusted for age, race, and education, were compared between metabolically healthy and at risk obese. Nearly 1 in 5 (149/775, or 19\%) of obese American INTERMAP participants were classified as metabolically healthy obese. Diet composition and most activity behaviors were similar between obesity phenotypes, although metabolically healthy obese women reported higher sleep duration than at risk obese women. These results do not support hypotheses that diet composition and/or physical activity account for the absence of cardiometabolic abnormalities in metabolically healthy obese.

\section{INTRODUCTION}

\begin{abstract}
Obesity is associated with higher mortality risks, higher health care costs, impaired physical functioning, lower quality of life, and higher morbidity from major cardiovascular and noncardiovascular causes. ${ }^{1-6}$ Obesity often clusters with other major cardiovascular disease (CVD) risk factors including prehypertension and hypertension, dyslipidemia, and impaired glucose tolerance ${ }^{7,8}$, yet a subset of the obese appears to be protected from these risks. Two obesity phenotypes have been described, 1) "metabolically healthy" obese and 2) "at risk"
\end{abstract}

Users may view, print, copy, and download text and data-mine the content in such documents, for the purposes of academic research, subject always to the full Conditions of use:http://www.nature.com/authors/editorial_policies/license.html\#terms

Corresponding author: Arlene Hankinson, MD, MS, Department of Preventive Medicine, Northwestern University, 680 N Lake Shore Drive, Suite 1400, Chicago, IL 60611, Phone: (312) 503-1065; Fax: (312) 908-9588. alhankinson@northwestern.edu. 
obese $^{9-11}$. The metabolically healthy obese phenotype is broadly defined as body mass index (BMI) $\geq 30 \mathrm{~kg} / \mathrm{m}^{2}$ and favorable levels of blood pressure, lipids, and glucose.

According to data from the 1994-2004 National Health and Nutrition Examination Survey

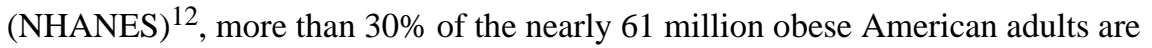
metabolically healthy obese, defined as obese with no more than one cardiometabolic risk factor. With metabolically healthy defined as no adverse levels of cardiometabolic risk factors, $17 \%$ of obese are metabolically healthy.

Determinants of obesity phenotypes are unclear, particularly role of diet composition, i.e., intake measured either by food groups (food-based categories) or nutrients (macro-/ micronutrients); and behaviors related to activity levels, i.e., sleep duration, television viewing time, other sedentary behavior, and physical activity ${ }^{13}$. To our knowledge, there are no population-based data on diet composition by obesity phenotype; aside from leisure time activity, behaviors related to activity levels have rarely been assessed in obesity phenotypes. Furthermore, obese phenotypes have rarely been investigated in ethnically diverse groups, including those with inordinately high prevalence rates of obesity and CVD risk factors. Here we present data on these matters for a multi-ethnic cohort of 2,195 adults ages 40-59 years from eight diverse U.S. population samples of the INTERMAP Study ${ }^{14-16}$. We hypothesized that metabolically healthy obese have more favorable behavioral factors, including less adverse diet composition and higher physical activity levels than at risk obese.

\section{METHODS AND PROCEDURES}

\section{Participants}

The International Population Study on Macro/Micronutrients and Blood Pressure (INTERMAP) is a cross-sectional investigation of the relation of dietary factors (foods/ nutrients) and urinary metabolites to blood pressure ${ }^{14,16,17}$. It includes 4,680 men and women ages 40-59 from 17 diverse population samples, including 2,195 persons from eight diverse American population samples. Details about eligibility criteria and baseline demographic characteristics have been published ${ }^{16}$. Each sample was selected randomly from an age/gender stratified population list, to give approximately equal numbers of people in each of four 10-year age-gender groups (men ages 40-49 and 50-59; women ages 40-49 and 50-59).

This study is based on 2,195 participants from US samples. Of these, non-obese persons $\left(\mathrm{BMI}<30 \mathrm{~kg} / \mathrm{m}^{2}\right.$ ) were excluded, leaving a total of 775 obese American adults (398 men and 377 women), i.e., $35 \%$ of the American INTERMAP cohort, for analysis.

\section{Data collection}

Each participant attended the local INTERMAP research center on four occasions: two visits on consecutive days with a further two visits on consecutive days on average three weeks later. Whenever possible, one visit by each participant included a weekend day (or an equivalent rest day) according to work schedule. All data were collected by trained and certified staff using high quality standardized methods. The protocol was approved by the 
institutional review board at each research center and written consent was obtained from each participant.

Blood pressure and other data-Blood pressure of the seated participant was measured twice per visit (four different days) with a random zero sphygmomanometer after emptying the bladder and at least five minutes of rest in a quiet room. Pulse was measured three times per visit. At the first and third visit, height without shoes was measured using a vertically placed rule with the base at floor level and weight was measured using a balance beam or weighing scale. BMI was calculated as weight divided by height squared $\left(\mathrm{kg} / \mathrm{m}^{2}\right)$. Data on demographic and other factors, including education, sleep duration, television viewing time, leisure-time and work-related physical activity (usual hours per day spent sitting or doing light and moderate/heavy activity) smoking (current, former, or never smoker), family and previous medical history, current special diet, and medication use were collected by interviewer-administered questionnaire.

Dietary data-Dietary data were collected at each of the four visits by a trained certified interviewer using the in-depth multipass 24-hour recall method. All foods and beverages consumed in the previous 24 hours were recorded. To aid accurate recall, fresh foods of varied standardized portion sizes, food and drink models, containers of various types and sizes, and photographs were used. Interviewers used neutral probing techniques to check for completeness of items reported, and details such as brand names of foods, quantities, processing methods, additions in cooking and/or at table, and amounts left on plate ${ }^{15,16}$. Dietary information was directly computerized with use of a program to guide on-screen coding. Nutrient intakes of participants were calculated from U.S. specific food tables. Daily alcohol consumption (amount and type of alcoholic beverage) over the previous seven days and, for abstainers, information on previous drinking were obtained by interview twice, at the first and third visits; these data were in addition to those on alcohol intake from the four 24-hour dietary recalls. Abstainers were those who reported no current alcohol consumption in the four dietary recalls, all others were considered current drinkers. 83 nutrient variables, including total energy intake and macro-/micronutrients were assessed. Food group analyses were performed using the Nutrition Data System for Research (NDSR) version 2.91from the Nutrition Coordinating Center (NCC) at the University of Minnesota ${ }^{18}$. The NDSR includes 9 generic food groups and 168 subgroups that were considered in these analyses. Based on NDSR groupings, INTERMAP investigators created 16 food groups and 42 food-based subgroups, concentrating on specific food subgroups including meat and fish, dairy, eggs, fruits, vegetables, and grains. The 34 nutrient variables and 14 food group variables selected for this analysis were those putatively associated with healthy diet composition. The food group variables are described in Appendix A. Both individual nutrient and food group variables were analyzed in regard to the outcomes of interest. 19, 20

Urinary data-For the optimal assessment of dietary sodium, potassium, and total protein intake, two timed 24-hour urine specimens were collected; urinary sodium (Na), potassium (K), creatinine, urea (index of 24-hour total protein intake), and multiple other metabolites were measured ${ }^{16}$. Timed collections were started at the research center on the first and third visits, and completed at the center the following day. Urine aliquots were stored frozen at 
$-20^{\circ} \mathrm{C}$ before being shipped frozen to a Central Laboratory in Leuven, Belgium, where analyses were performed with extensive internal and external quality control; further analyses were subsequently done at a Central Laboratory in London, England. Individual excretion values were calculated as the product of concentrations in the urine and urinary volume corrected to 24 hours. The average of the two excretion values was used.

\section{Definition of obesity phenotypes}

Since there are no uniform criteria to define obese phenotypes, an INTERMAP specific definition was developed. Metabolically healthy was defined as meeting all of the following criteria: favorable blood pressure ( $₫ 20 / \$ 80 \mathrm{~mm} \mathrm{Hg}$ ) and no medication or special diet for hypertension; no physician diagnosis, medication, or special diet for other metabolic risk factors (i.e., diabetes and dyslipidemia); no prevalent cardiovascular disease. Any participant not meeting these criteria was classified as at risk obese.

\section{Statistical analyses}

Prevalence rates of metabolically healthy obese and at risk obese were calculated separately for the two genders and for the whole sample. Overall mean values for continuous descriptive variables (age, years of education, BMI, pulse) and proportions of categorical descriptive variables ( $\%$ female, $\%$ nonWhite, $\%$ current drinkers, $\%$ current smokers, $\%$ with family history of hypertension) were calculated for metabolically healthy and at risk obese. Differences in overall mean values between the metabolically healthy and at risk obese were compared using t-tests; proportions were compared using chi-square analyses. All other analyses were done separately within gender groups. Means, adjusted for age, race, and education, were calculated for 14 food groups, 34 macro-/micronutrients, and 5 activity behavior variables. Data for food group variables were not normally distributed; adjusted means of log-transformed food group values were calculated and back transformed for presentation as geometric means. T-tests were used to compare adjusted means of metabolically healthy and at risk obese by gender. To address potential type I error from multiple comparisons of the 53 food group, nutrient, and activity variables, Bonferroni correction was performed; the corrected a level for significant results was $0.05 / 53=0.001$. In a sensitivity analysis, all of the aforementioned analyses were repeated with the criteria for healthy obese also including nonsmoking. Analyses were done by the first author (A. Hankinson) with SAS statistical software, version 9.2 (SAS Inc, Cary, NC).

\section{RESULTS}

\section{Prevalence and characteristics of obese phenotypes}

Descriptive statistics for 775 obese American INTERMAP participants, stratified by gender, are shown in Table 1; 19\% (149/775) met the definition of metabolically healthy obese. Prevalence of metabolically healthy obese was similar in men and women (20\% and 19\%, respectively). Compared with at risk obese, metabolically healthy obese adults were significantly younger; there were the following nonsignificant differences: lower BMI, higher proportion of nonWhite race/ethnicity (Black, Hispanic, Asian, and Native American), and lower prevalence of family history of hypertension. In sensitivity analysis with no smoking as an additional criterion for metabolically healthy obese, 127 of the 775 
participants (16\%) met all criteria; the remaining 648 were considered at risk obese. Results (not tabulated here) were nearly identical to the overall results.

\section{Diet composition: Food groups and macro-/micronutrients}

Fourteen food groups and 34 dietary macro-/micronutrients were compared between at risk and metabolically healthy obese phenotypes. There were no significant differences in food groups (Table 2) or nutrients (Table 3 ) between obesity phenotypes. In both genders, metabolically healthy obese reported nonsignificantly lower energy intake compared with at risk obese. There was no significant difference in type or amount of food group intake, including fruits and vegetables, grains, meats, fish, and sugar sweetened beverages across obesity phenotypes. In women, the metabolically healthy obese reported higher amounts of vegetable protein and starch and lower amounts of total protein (estimated from urinary urea) compared with at risk obese. These differences had p-values below 0.05, but did not meet the alpha level of significance adjusted for multiple comparisons. Intakes of other macro-/micronutrients, including total fat, protein, carbohydrate, fiber, iron, and alcohol were similar across the two obesity phenotypes for men and women.

\section{Activity behaviors: Sleep, television viewing time, other sedentary activity, physical activity levels}

There were no significant differences in sleep duration, television viewing time, other sedentary activity, and physical activity levels between obesity phenotypes in men (Table 4). In women, metabolically healthy obese reported significantly higher sleep duration than at risk obese ( 7.6 hours/day vs. 7.0 hours/day); there were no significant differences in other activity behaviors.

\section{DISCUSSION}

In this population-based study of 775 obese American middle-aged adults, 19\% (nearly 1 in 5) met criteria for metabolically healthy obese, defined by favorable levels of cardiometabolic risk factors. This study is the first, to our knowledge, to compare diet composition between obesity phenotypes. Diet composition, measured as intake of food groups and macro-/micronutrients, and activity behaviors (e.g., television viewing time, other sedentary activity, and physical activity levels) were similar between obesity phenotypes, with the exception of longer sleep duration in metabolically healthy obese women. Other factors, including BMI, energy intake, and sociodemographic characteristics were similar between obesity phenotypes. These results prevailed with no smoking as an additional criterion for metabolically healthy obese. Our results indicate that diet composition and activity behaviors do not explain the absence of cardiometabolic abnormalities in metabolically healthy obese adults.

The $19 \%$ prevalence rate for the metabolically healthy obese phenotype observed in the present study is lower than the 23-32\% reported in previous studies. ${ }^{11,12,21}$ Our lower prevalence rate may be explained by our more strict definition for the metabolically healthy obese phenotype, particularly our criteria for favorable blood pressure ( $\leq 120 / 80 \mathrm{~mm} \mathrm{Hg}$ and no antihypertensive medications). Other studies with blood pressure in the definition of 
metabolically healthy obesity used a threshold of $140 / 90$ or $130 / 85 \mathrm{mmHg}$, i.e., inclusive of persons with prehypertension ${ }^{22}$ in the metabolically healthy obese group. Also, some definitions included one cardiometabolic abnormality in the definition of the metabolically healthy obese phenotype. A recent NHANES report estimated the prevalence of metabolically healthy American obese to be $32 \%$ based on a definition including one cardiometabolic abnormality; prevalence was $17 \%$ when metabolically healthy was defined as absence of any cardiometabolic abnormalities, i.e., a rate similar to ours. ${ }^{12}$

No significant associations were observed between obesity phenotypes and diet composition. This absence of significant association between diet composition and obesity phenotypes is relevant in the light of compelling observational study and clinical trial evidence that a healthy diet may be associated with the metabolically healthy obese phenotype regardless of diet quantity (energy intake). For example, in overweight/obese adults, the Diabetes Control and Complications Trial and the Finnish Diabetes Prevention Study both reported a $58 \%$ reduction in incident diabetes through diet and activity interventions with modest $(<10 \%)$ weight loss. ${ }^{23,} 24$ The OmniHeart trial demonstrated beneficial effects on blood pressure, high density lipoprotein cholesterol (HDL-C), and triglycerides with isocaloric replacement of carbohydrates by proteins or unsaturated fat in adults with mean BMI of $30.2 \mathrm{~kg} / \mathrm{m}^{2}{ }^{25}$ INTERMAP and other observational studies have shown similar associations between diet composition and CVD risk factor levels. In particular, prior work in the INTERMAP cohort has demonstrated an inverse relationship to blood pressure of several macro-/micronutrients, including calcium, magnesium, phosphorous, total and non heme iron, vegetable protein, and omega- 3 fatty acids. ${ }^{26-31}$ Of these nutrients, in the present study only vegetable protein was noted to be higher in the metabolically healthy obese; the finding prevailed only in women and the p-value was not statistically significant after adjustment for multiple comparisons.

While no study, to our knowledge, has reported sleep duration or sedentary behaviors in obesity phenotypes, a few cross-sectional studies have investigated associations between physical activity levels and obesity phenotypes, with contradictory results. Self-reported activity data showed associations between higher activity and the metabolically healthy obesity phenotype, while objective physical activity data did not. ${ }^{12,32}$ Results of the current study for activity levels are concordant with objective physical activity data. Among obese women, the metabolically healthy reported 0.6 hours longer sleep duration than at risk obese. Although shorter sleep duration has often been associated with obesity in crosssectional and prospective studies, ${ }^{33,34}$ this is the first report of an association with the at risk obesity phenotype. It is hypothesized that shorter sleep duration and obesity may be linked through neuroendocrine changes that influence appetite and favor a positive caloric balance, ${ }^{34}$ or through behaviors often correlated with short sleep duration, such as television viewing time and consumption of high energy foods. ${ }^{35,36}$ These hypotheses are not likely to explain the observed association with obesity phenotypes in our cohort of American INTERMAP women, since energy intake, television viewing time, and diet composition were similar in at risk and metabolically healthy obese women. While measures of sedentary behavior were not statistically different between obesity phenotypes, metabolically healthy obese men reported fewer hours of television viewing time and metabolically healthy obese 
women reported less sedentary activity than their at risk obese counterparts, suggesting less sedentary behavior in the metabolically healthy obese compared with at risk obese.

Mean age of metabolically healthy obese was nearly three years younger than the at risk obese, consistent with findings from NHANES. ${ }^{12}$ Other studies comparing mean age between obesity phenotypes reported discrepant findings, showing significantly older age of healthy obese, ${ }^{12,37}$ or no significant difference in age..$^{38-41}$

Our study has several strengths, including our strict definition of metabolically healthy obese and the high quality diet data, meticulously measured with four separate in-depth multi pass 24-hour recalls. We also recorded several measures of activity behavior, including sleep duration, two measures of sedentary behavior, and physical activity levels of varying intensities (light and moderate to vigorous). We corrected for multiple testing using a conservative method to adjust the alpha level, which likely inflated type II (false negative) error. Our study is limited by its cross sectional design, which precludes assessment of relationships between antecedent behavioral factors and obesity phenotypes. Another limitation needing emphasis is the absence of INTERMAP data on blood glucose and lipids for our obesity phenotype classification. Finally, although diet data was collected with rigor, recall bias of dietary data is likely.

In conclusion, nearly 1 in 5 (19\%) of obese INTERMAP middle-aged American adult participants were classified as metabolically healthy, a prevalence rate similar to that of previous reports using similar definitions. Diet composition, measured by food group and macro-/micronutrient intake, was not associated with obesity phenotype. While sleep duration was associated with obesity phenotype in women, other activity behaviors factors, including television viewing time, other sedentary activity, and physical activity levels were not significantly related to obesity phenotype. These results do not support hypotheses that diet composition and physical activity account for absence of cardiometabolic abnormalities in metabolically healthy obese adults.

\section{Acknowledgments}

This research was supported by grant 2R01HL50490 from the National Heart, Lung, and Blood Institute, National Institutes of Health. It is a pleasure to express appreciation to all INTERMAP staff at local, national, and international centers for their invaluable efforts; a partial listing of these colleagues is given in reference 14 of this article.

\section{References}

1. Poirier P, Giles TD, Bray GA, et al. Obesity and cardiovascular disease: pathophysiology, evaluation, and effect of weight loss: an update of the 1997 American Heart Association Scientific Statement on Obesity and Heart Disease from the Obesity Committee of the Council on Nutrition, Physical Activity, and Metabolism. Circulation. 2006; 113:898-918. [PubMed: 16380542]

2. Fontaine KR, Barofsky I. Obesity and health-related quality of life. Obes Rev. 2001; 2:173-82. [PubMed: 12120102]

3. Jensen GL. Obesity and functional decline: epidemiology and geriatric consequences. Clin Geriatr Med. 2005; 21:677-87. v. [PubMed: 16182081]

4. Daviglus ML, Liu K, Yan LL, et al. Relation of body mass index in young adulthood and middle age to Medicare expenditures in older age. Jama. 2004; 292:2743-9. [PubMed: 15585734] 
5. Fontaine KR, Redden DT, Wang C, Westfall AO, Allison DB. Years of life lost due to obesity. Jama. 2003; 289:187-93. [PubMed: 12517229]

6. Pi-Sunyer FX. Health implications of obesity. Am J Clin Nutr. 1991; 53:1595S-603S. [PubMed: 2031492]

7. Reaven GM. Insulin resistance: the link between obesity and cardiovascular disease. Endocrinol Metab Clin North Am. 2008; 37:581-601. vii-viii. [PubMed: 18775353]

8. Abbasi F, Brown BW Jr, Lamendola C, McLaughlin T, Reaven GM. Relationship between obesity, insulin resistance, and coronary heart disease risk. J Am Coll Cardiol. 2002; 40:937-43. [PubMed: 12225719]

9. Ferrannini E, Natali A, Bell P, Cavallo-Perin P, Lalic N, Mingrone G. European Group for the Study of Insulin Resistance (EGIR) . Insulin resistance and hypersecretion in obesity. J Clin Invest. 1997; 100:1166-73. [PubMed: 9303923]

10. Karelis AD, St-Pierre DH, Conus F, Rabasa-Lhoret R, Poehlman ET. Metabolic and body composition factors in subgroups of obesity: what do we know? J Clin Endocrinol Metab. 2004; 89:2569-75. [PubMed: 15181025]

11. Meigs JB, Wilson PW, Fox CS, et al. Body mass index, metabolic syndrome, and risk of type 2 diabetes or cardiovascular disease. J Clin Endocrinol Metab. 2006; 91:2906-12. [PubMed: 16735483]

12. Wildman RP, Muntner P, Reynolds K, et al. The obese without cardiometabolic risk factor clustering and the normal weight with cardiometabolic risk factor clustering: prevalence and correlates of 2 phenotypes among the US population (NHANES 1999-2004). Arch Intern Med. 2008; 168:1617-24. [PubMed: 18695075]

13. Wildman RP. Healthy obesity. Curr Opin Clin Nutr Metab Care. 2009; 12:438-43. [PubMed: 19474713]

14. Beevers DG, Stamler J. Background to the INTERMAP study of nutrients and blood pressure. Journal of Human Hypertension. 2003; 17:589-90. [PubMed: 13679949]

15. Dennis B, Stamler J, Buzzard M, et al. INTERMAP: the dietary data--process and quality control. Journal of Human Hypertension. 2003; 17:609-22. [PubMed: 13679951]

16. Stamler J, Elliott P, Dennis B, et al. INTERMAP: background, aims, design, methods, and descriptive statistics (nondietary). Journal of Human Hypertension. 2003; 17:591-608. [PubMed: 13679950]

17. Elliott P, Stamler R. Manual of operations for "INTERSALT", an international cooperative study on the relation of sodium and potassium to blood pressure. Control Clin Trials. 1988; 9:1S-117S. [PubMed: 3396367]

18. Nutrition Data System for Research. [Accessed May 23, 2011] Appendix 10 http:// www.ncc.umn.edu/products/ndsrmanual2010append10.pdf

19. Appel LJ, Moore TJ, Obarzanek E, et al. A clinical trial of the effects of dietary patterns on blood pressure. DASH Collaborative Research Group. N Engl J Med. 1997; 336:1117-24. [PubMed: 9099655]

20. Harsha DW, Lin PH, Obarzanek E, Karanja NM, Moore TJ, Caballero B. Dietary Approaches to Stop Hypertension: a summary of study results. DASH Collaborative Research Group. J Am Diet Assoc. 1999; 99:S35-9. [PubMed: 10450292]

21. Iacobellis G, Ribaudo MC, Zappaterreno A, Iannucci CV, Leonetti F. Prevalence of uncomplicated obesity in an Italian obese population. Obes Res. 2005; 13:1116-22. [PubMed: 15976155]

22. Chobanian AV, Bakris GL, Black HR, et al. The Seventh Report of the Joint National Committee on Prevention, Detection, Evaluation, and Treatment of High Blood Pressure: the JNC 7 report. Jama. 2003; 289:2560-72. [PubMed: 12748199]

23. The Diabetes Control and Complications Trial Research Group. The effect of intensive treatment of diabetes on the development and progression of long-term complications in insulin-dependent diabetes mellitus. N Engl J Med. 1993; 329:977-86. [PubMed: 8366922]

24. Tuomilehto J, Lindstrom J, Eriksson JG, et al. Prevention of type 2 diabetes mellitus by changes in lifestyle among subjects with impaired glucose tolerance. N Engl J Med. 2001; 344:1343-50. [PubMed: 11333990] 
25. Appel LJ, Sacks FM, Carey VJ, et al. Effects of protein, monounsaturated fat, and carbohydrate intake on blood pressure and serum lipids: results of the OmniHeart randomized trial. Jama. 2005; 294:2455-64. [PubMed: 16287956]

26. Stamler, J. Improved Nutrition: Key to Solving the Populationwide Blood Pressure Problem. In: Mancini, M., editor. Nutritional and Metabolic Bases of Cardiovascular Disease. Vol. 2011. Wiley-Blackwell; United Kingdom: 2011.

27. Kesteloot Dagger H, Tzoulaki I, Brown IJ, et al. Relation of urinary calcium and magnesium excretion to blood pressure: the international study of macro- and micro-nutrients and blood pressure and the international cooperative study on salt, other factors, and blood pressure. Am J Epidemiol. 2011; 174:44-51. [PubMed: 21624957]

28. Elliott P, Kesteloot H, Appel LJ, et al. Dietary phosphorus and blood pressure: international study of macro- and micro-nutrients and blood pressure. Hypertension. 2008; 51:669-75. [PubMed: 18250363]

29. Elliott P, Stamler J, Dyer AR, et al. Association between protein intake and blood pressure: the INTERMAP Study. Arch Intern Med. 2006; 166:79-87. [PubMed: 16401814]

30. Tzoulaki I, Brown IJ, Chan Q, et al. Relation of iron and red meat intake to blood pressure: cross sectional epidemiological study. Bmj. 2008; 337:a258. [PubMed: 18632704]

31. Ueshima H, Stamler J, Elliott P, et al. Food omega-3 fatty acid intake of individuals (total, linolenic acid, long-chain) and their blood pressure: INTERMAP study. Hypertension. 2007; 50:313-9. [PubMed: 17548718]

32. Brochu M, Tchernof A, Dionne IJ, et al. What are the physical characteristics associated with a normal metabolic profile despite a high level of obesity in postmenopausal women? J Clin Endocrinol Metab. 2001; 86:1020-5. [PubMed: 11238480]

33. Chaput JP, Despres JP, Bouchard C, Tremblay A. Short sleep duration is associated with reduced leptin levels and increased adiposity: Results from the Quebec family study. Obesity (Silver Spring). 2007; 15:253-61. [PubMed: 17228054]

34. Chaput JP, Despres JP, Bouchard C, Tremblay A. The association between sleep duration and weight gain in adults: a 6-year prospective study from the Quebec Family Study. Sleep. 2008; 31:517-23. [PubMed: 18457239]

35. Shi Z, McEvoy M, Luu J, Attia J. Dietary fat and sleep duration in Chinese men and women. Int J Obes (Lond). 2008; 32:1835-40. [PubMed: 18982012]

36. Westerlund L, Ray C, Roos E. Associations between sleeping habits and food consumption patterns among 10-11-year-old children in Finland. Br J Nutr. 2009; 102:1531-7. [PubMed: 19664303]

37. Jennings CL, Lambert EV, Collins M, Joffe Y, Levitt NS, Goedecke JH. Determinants of insulinresistant phenotypes in normal-weight and obese Black African women. Obesity (Silver Spring). 2008; 16:1602-9. [PubMed: 18421268]

38. Marini MA, Succurro E, Frontoni S, et al. Metabolically healthy but obese women have an intermediate cardiovascular risk profile between healthy nonobese women and obese insulinresistant women. Diabetes Care. 2007; 30:2145-7. [PubMed: 17507694]

39. Karelis AD, Faraj M, Bastard JP, et al. The metabolically healthy but obese individual presents a favorable inflammation profile. J Clin Endocrinol Metab. 2005; 90:4145-50. [PubMed: 15855252]

40. Stefan N, Kantartzis K, Machann J, et al. Identification and characterization of metabolically benign obesity in humans. Arch Intern Med. 2008; 168:1609-16. [PubMed: 18695074]

41. Succurro E, Marini MA, Frontoni S, et al. Insulin secretion in metabolically obese, but normal weight, and in metabolically healthy but obese individuals. Obesity (Silver Spring). 2008; 16:1881-6. [PubMed: 18551117] 


\section{Table 1}

Descriptive statistics of at risk and metabolically healthy obese American adults, by gender - mean (standard deviation) or \% (n)

\begin{tabular}{|c|c|c|c|c|}
\hline \multirow[b]{2}{*}{ Variable } & \multicolumn{2}{|r|}{ Men $(n=398)$} & \multicolumn{2}{|c|}{ Women $(n=377)$} \\
\hline & At risk $(n=323)$ & Metabolically healthy $(n=75)$ & At risk $(\mathrm{n}=\mathbf{3 0 3})$ & Metabolically healthy $(n=74)$ \\
\hline Age, years* & $49.1(5.3)$ & $48.2(4.9)$ & $50.1(5.3)$ & $46.0(4.8)$ \\
\hline$\%$ non White, (n) & $45.8(148)$ & $44.0(33)$ & $54.8(166)$ & $63.5(47)$ \\
\hline Education, years & $14.7(2.8)$ & $15.1(2.8)$ & $13.7(2.8)$ & $13.6(2.9)$ \\
\hline Weight, $\mathrm{kg}$ & $106.6(16.4)$ & $103.2(13.2)$ & $95.5(16.3)$ & $90.1(12.3)$ \\
\hline Height, meters & $1.8(0.1)$ & $1.8(0.1)$ & $1.6(0.1)$ & $1.6(0.1)$ \\
\hline Body mass index, $\mathrm{kg} / \mathrm{m}^{2}$ & $34.6(4.4)$ & $33.1(3.3)$ & $36.4(5.4)$ & $35.0(4.4)$ \\
\hline Pulse, beats/minute & $75.4(10.2)$ & $72.0(9.6)$ & $75.7(9.7)$ & $76.4(7.6)$ \\
\hline Systolic blood pressure, $\mathrm{mmHg}^{*}$ & $126.7(11.6)$ & $112.2(5.0)$ & $126.2(13.5)$ & $109.7(6.2)$ \\
\hline Diastolic blood pressure, $\mathrm{mmHg}^{*}$ & $79.5(10.3)$ & $72.0(4.7)$ & $75.0(8.7)$ & $67.6(6.2)$ \\
\hline$\%$ Current drinkers, (n) & $73.1(236)$ & $81.3(61)$ & $57.4(174)$ & $58.1(43)$ \\
\hline$\%$ Current smokers, (n) & $16.1(52)$ & $13.3(10)$ & $13.9(42)$ & $16.2(12)$ \\
\hline$\%$ with family history of HTN, (n) & $68.1(220)$ & $57.3(43)$ & $76.6(232)$ & $68.9(51)$ \\
\hline
\end{tabular}

Abbreviations - HTN: hypertension

Metabolically healthy obese defined as BMI $\geq 30 \mathrm{~kg} / \mathrm{m}^{2}$ and meeting all of the following criteria: blood pressure $\unlhd 20 / \$ 0 \mathrm{~mm} \mathrm{Hg}$, no medication or special diet for hypertension, no physician diagnosis, medication, or special diet for cardiovascular disease (CVD) risk factors (i.e., diabetes and dyslipidemia),no prevalent CVD, and no special diet for weight loss or weight gain. Serum glucose and lipids were not measured.

*Values significantly different for at risk obese compared to metabolically healthy obese (p-value $<0.05)$ p-values calculated using t-test for means, chi-square test for proportions 

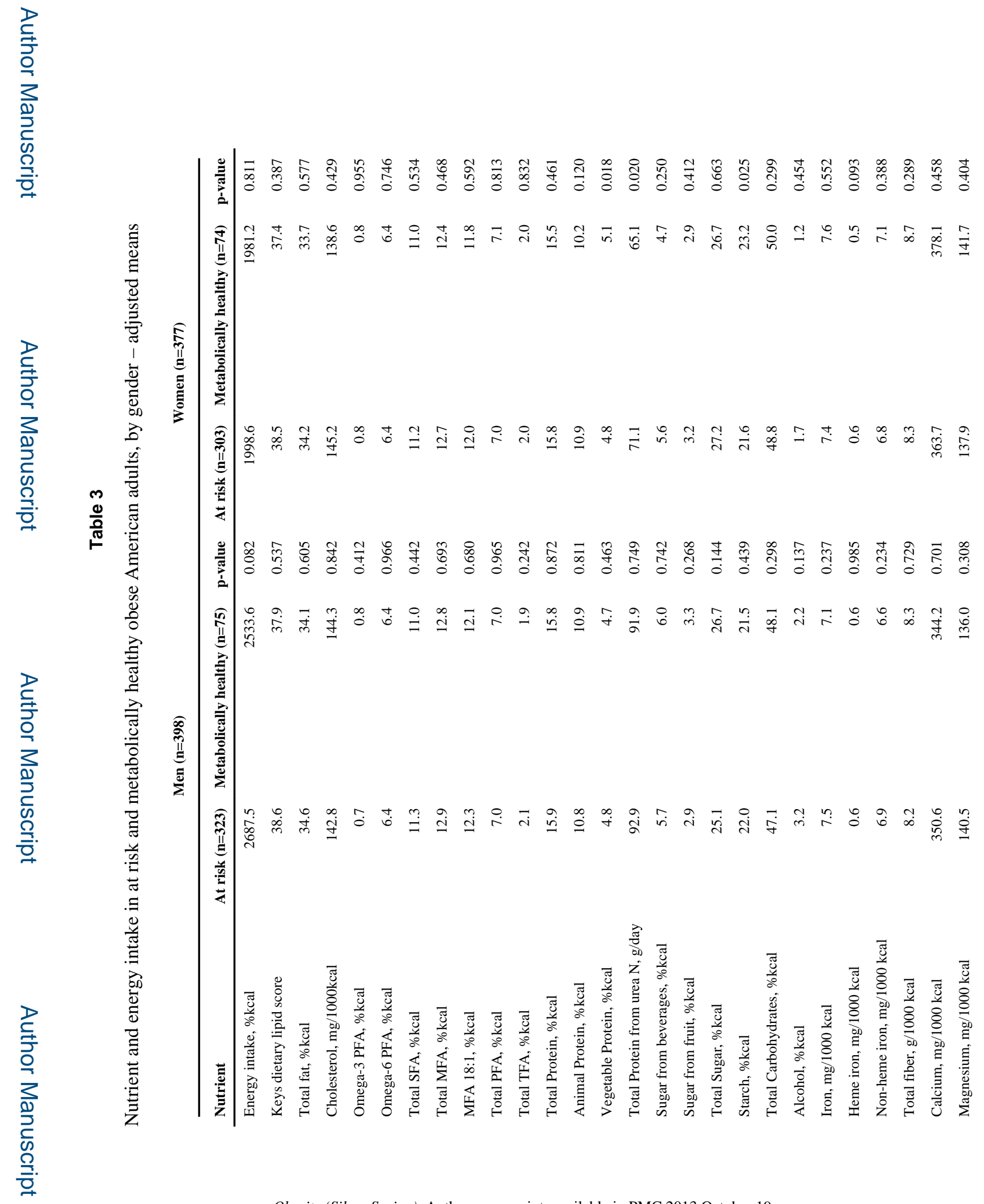

Obesity (Silver Spring). Author manuscript; available in PMC 2013 October 19. 


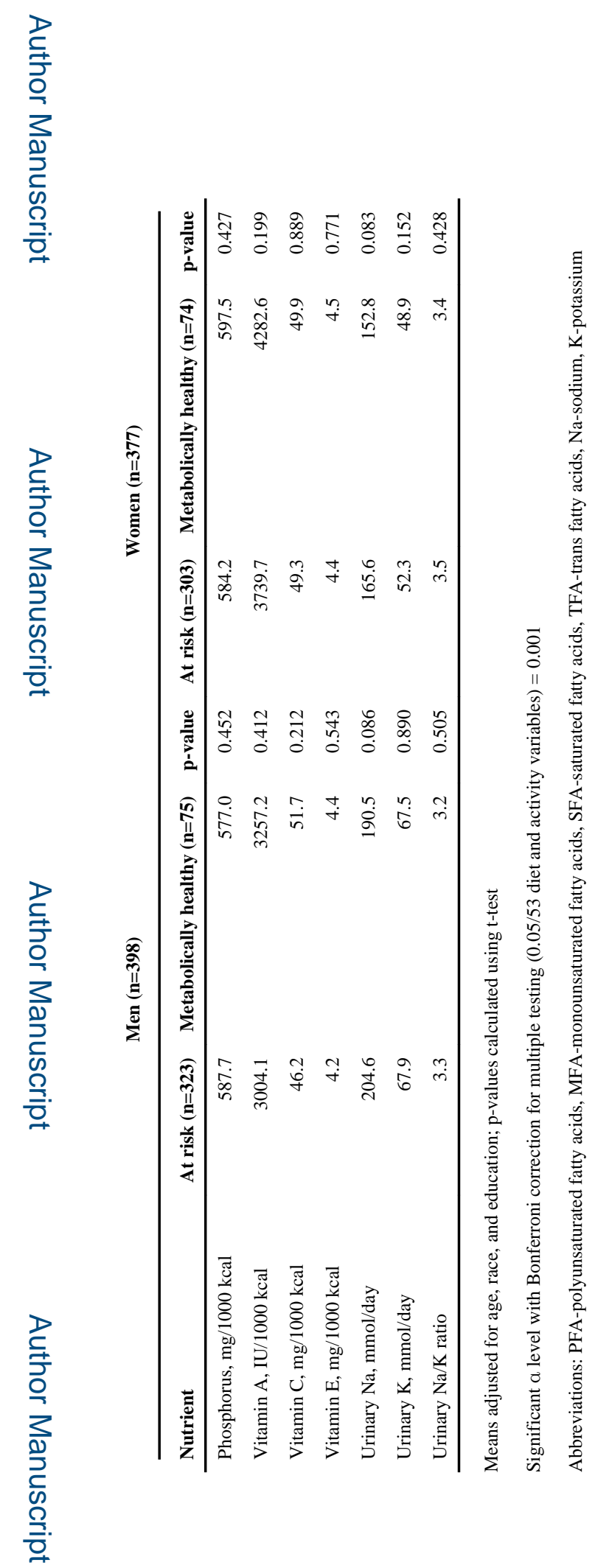

Obesity (Silver Spring). Author manuscript; available in PMC 2013 October 19. 


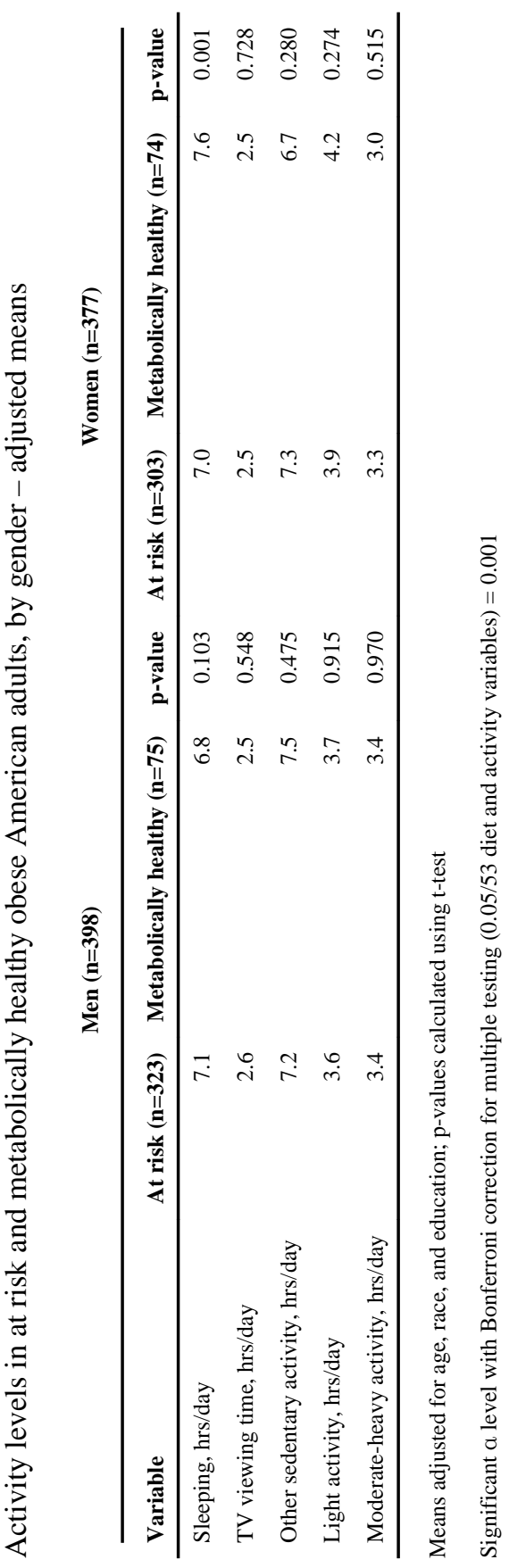

Obesity (Silver Spring). Author manuscript; available in PMC 2013 October 19. 


\section{Appendix A}

14 food group variables, adapted from Nutrition Data System for Research, Nutrition Coordinating Center University of Minnesota

\begin{tabular}{|c|c|}
\hline Food Groups and Subgroups & Description \\
\hline Total fruits & $100 \%$ fruit juices and drinks, sweetened and unsweetened fruits, dried fruits \\
\hline Fresh fruits & Subgroup of total fruits \\
\hline Total vegetables & Raw, cooked (fresh, frozen, or canned), vegetarian meat substitutes, vegetable recipes \\
\hline Total grains & Breads, rolls, biscuits, pancakes, ready to eat cereals, grains and flour \\
\hline Nuts, nut butters, and legumes & Nuts, nut butters, mature dried beans and peas \\
\hline Low fat dairy products & $\begin{array}{l}\text { Less than } 2 \% \text { fat content dairy products-i.e., cream, cheese, ice creams, milk/cheese recipes, milk, yogurt, } \\
\text { yogurt frozen, cocoa }\end{array}$ \\
\hline Higher fat dairy products & $2 \%$ or more fat content dairy products (listed above) \\
\hline Fish and seafood & Fish and fish roe, shellfish \\
\hline Poultry & Domestic and wild fowl \\
\hline Fresh meats & Beef, lamb, pork, veal, game \\
\hline Processed meats & Fresh and cured cold cuts, sausage \\
\hline Fats & Animal fats, margarines, table spreads, oils, shortening, salad dressing \\
\hline Sweets & Sugar, syrup, honey, jam, jelly, preserves, sweet sauces, candy \\
\hline Sugar sweetened beverages & Sweetened non-carbonated drinks $(<100 \%$ juice $)$, does not include artificially sweetened drinks \\
\hline
\end{tabular}

